\title{
Artificial Intelligence and Advances
}

\author{
Amrot Mitachew Agidew \\ Dunamis Social Entrepreneurs, Department of Computer Science, \\ Ethiopia
}

\author{
${ }^{*}$ Corresponding author \\ Amrot Mitachew Agidew, Dunamis Social Entrepreneurs, Department \\ of Computer Science, Ethiopia \\ Submitted: 26 Sept 2020; Accepted: 16 Sept 2020; Published: 22 Oct 2020
}

\begin{abstract}
Summary
This research abstract shares open source theme smart materials and technology for the Current and Future Global Challenges in relation with artificial intelligence which is the simulation of human intelligence processes by machines, especially computer systems. AI will also have a major impact on illegal/illicit/legal harmful drugs, chemicals, toxic herbs and others Control Intervention.
\end{abstract}

the patent pending this scientific computing innovation; will include illegal/illicit/legal harmful drugs, chemicals, toxic herbs and others Control security system and special purpose computer connected intelligent equipment's, with sensors capable of taking thousands of measurements throughout the production process and generating billions of data points used to monitor, analyze and control the trafficking process.

AI and machine learning are also contributing to the development of next-generation security system, accelerating the development of security intervention for conditions where there are no viable options today.

Artificial intelligence promises both to improve existing goods and services, by enabling the automation of many tasks, to greatly increase the efficiency with which they are produced. But it may have an even larger impact on the economy by serving as a new general-purpose method of invention for unique or special tasks; Where Artificial intelligence (AI) clearly lead to better outcomes, already producing benefits and optimizing processes, increasingly sophisticated algorithms and machine learning techniques of data on a particular issue, generate insights, detections and resulting with more efficiency than teams of humans ever could.

Keywords: Artificial, Automations, Efficiency, Generation, Intelligence, Purpose, Sensors, Systems, Unique, Viable.

\section{Introduction}

Artificially intelligent advanced smart materials are the basis of many applications, including sensors, actuators or muscles and artificially intelligent technology products, particularly as electro active polymers (EAPs).

Artificial intelligence is transforming every aspect of our lives, from the way we work to the way we shop and socialize. This technology has the potential to deliver incredible benefits to society, increasing efficiency, improving safety and quality of life, transforming health care, and delivering new capabilities that we cannot even imagine. This research study can manage the risks, disruptions and fears of artificial intelligence to maximize its benefits to our economy and society. But, the above Both Disciplines Build Security Which is a broad term in industry and government. There is a myriad of security contexts on a variety of levels from the individual to nationwide.
Artificial intelligence and machine learning technologies are being applied and developed across this spectrum. While many of these technologies have the potential and have greatly benefited society (helping reduce The Manual Illegal and Illicit Substances Abuse, for example) the evolving social contexts and applications of these technologies often leave more questions than answers, in terms of rules, regulations and moral judgments in their wake. Artificial intelligence and security were in many ways made for each other, and the modern approaches of machine learning seem to be arriving just in time to fill in the gaps of previous rule based security systems.

The purpose of this article is to shed light on current trends and applications, in industry and government, at the intersection of Smart Materials, artificial intelligence and the security field. In addition to a spotlight on current uses (by no means inclusive), we also touch on up and coming applications and room for innovation (triggered by evolving needs of individuals and the larger population). 
I've referenced several of my interviews with researchers and practitioners in this space, and their insights and experiences are responsible for some of the applications explored in this article. An overarching theme in their varied responses points to an important implication using artificial intelligence to stay at least one step ahead of attackers, errors, and system failures. It's important to emphasize that as threats and social contexts evolve, so too will the technology need to adapt as well as the rules and regulations that govern the use of such technologies.

The potential future Featured application is meant to spark ideas about some of the directions in which AI technologies are headed and also illuminate a handful of key obstacles, challenges that need to be reconciled before the technology can begin to reach its full potential.

This Digital Illegal and Illicit Substances detection and Sensoring typically involve the collection of large scale Bio Information's to Address public health Strategy and policy. Some methods and models might be aimed at making inferences about unique Substances that could drive actions, such as alerting under This Security System's Controller, to improve individual or public health outcomes; this idea, while done with good intent, can undermine a basic goal of many Drug Abuse law to allow individuals to control who knows what about them. To infer health conditions and risks from non-medical interventions, whether through Bio informational or Biometric Control contexts. This provides ample opportunity for reflection on directions in balancing innovation and regulation. Although much Bio data are already Programmed or on online and machine learning has the potential capability to predict Nation citizen's future wellbeing status from health-related to other forms of information, this document emphasizes the point that our country does not currently have the laws, customs, culture or mechanisms to enable society to benefit from these types of proposed innovations currently. Perhaps the most important innovation in this arena is developing good policy and strategy i.e. how to use develop an infrastructure and efficient system, rather than the manual ineffective machine learning technologies used to detect non illegal and illicit substances information.

The airport security worldwide has to reformulated by Dynamic Aviation Risk Management Solution, the smart system will integrate illegal and illicit substances information across the aviation personalized security profile for each person on a flight basis. Currently AI simulated and supported security systems have in efforts of through pending projects like these security system development, where the next steps are to move from simulation to real investigations that utilize this type of tools in machine human collaborations.

About ethics and rules and regulations will stay strong, it seems likely that smart materials and artificial intelligent technologies able to detect these type of crimes in the making and alert authorities will be used in the future to reinforce security at major security lines or crossways such as educational institutions, ports, airports, industrial facilities, prisons and other societies.
It's worth emphasizing that AI sensor tools will be introduced in this project with the proper scientific research development and regulations in place have the potential to lessen or remove human bias as opposed to substantiating its effects.

Recent breakthroughs in machine learning and artificial intelligence (A.I.) have prompted breathless speculation about nationwide security applications. Yet most of that work has focused narrowly on illegal and illicit substances control and recovery interventions implications for smart material or systems, rather than on the broader security environment, Apart from A.I might concerned with in the core questions of international relations and foreign policy.

The Political scientists, researchers, scholars and intellectuals have speculated about this security system ever since it was first developed and promised to resolve the puzzled international relations of over a disturbing possibility. The general capabilities of this security system are integrating it with the wireless sensor network from under the radar based satellite wireless connectivity.

Artificial intelligence somehow introduces some Forms of uncertainty. But exactly i am enabling those circumstances to be used as nonoperational over the powerful functionalities. The prospect that a rival power might use an inspiring A.I and manufactures this security system's in innovative or scientific and unexpected ways is enough to exacerbate existing security concerns.

\section{Materials and Methods}

Machine Intelligence (MI) is poised to create profound changes to our lives, job and economy. In breadth and scale of impact, MI will rival or exceed past technological revolutions while potentially altering centers of economic power and regional and global influence. Many countries are already making significant policy decisions to prepare for the impact of MI and are investing in MI research and development to outpace allies and competitors across economic and national security dimensions of illegal and illicit substances. If the United States wants to avoid being left behind in the "MI Revolution for illegal and illicit substances or illegal migration" countries need to develop a coordinated international strategy that covers research and development, industrial specialization, and the social impacts of these security system technologies. This report offers a framework for determining which guiding principles should shape U.S. policy in response to the growth of MI applications in defense, education, health care, and the economy. The authors offer recommendations for how the United States can maintain a competitive advantage in MI and navigate the risks and challenges associated with it. Smart or intelligent materials are material that has to respond to stimuli and environmental changes and to activate their function according these changes.

The stimuli like a light, Electrical, Chemical and mechanical, etc. can originate internally or externally Smart materials and related technologies have been drawing an increasing amount of attention from researchers in related fields worldwide. 
This Control devices Self detection or diagnostic technique is Self-corrective, self-controlled, self-healing. The aim of this component is to collect the raw data needed for an appropriate sensing and monitoring of the composition or structure. The purpose of these parts is to forward the raw data to the local and or central command and control units.

Command and control unit or brain have the role of these unit is to manage and control the whole system by analyzing the data reaching the appropriate conclusion and determining the actions requires.

\section{Results}

A smart material is one that exhibits some observable effect in one domain when stimulated through another domain. These cover all domains including mechanical, electrical, chemical, and thermal and so on. Smart materials can add new capabilities to artificially intelligent technology products and especially this illegal and illicit substances control sensor scientific innovation. Can use biodegradable, biocompatible and selectively dissolvable polymers. Smart materials largely cover the same set of physical properties (stiffness, elasticity, viscosity) as state of the technologies that have the potential to deliver this capability. Smart materials can be divided into three group's hydraulic and pneumatic soft systems; smart actuator and sensor materials and stiffness changing materials.

How will this security system appear in our lives and how will we interact with the security line and adapt it; can foresee smart object, communicate and integration on the electronic components [1-3].

\section{Acknowledgement}

Often the nature of the first contact between individuals and institutions sets the tone and speed in which their relationships will subsequently evolve. Human assets such as, the patience to listen, capacity to take fundamentally innovative decisions and, determination to act in a timely manner, are essential in order to make certain things happen in a world of new challenges and uncertainties. Especial acknowledgement belongs to the pivotal contribution made by national archive and library agency. I thank, express my loyalty and affiliation to our country's wise leadership headed by His highest Excellency Cornell, Dr. Abiy Ahmed Ali, prime minister of FDRE "long live", His higher Excellency commissionaire, Dr. Workineh Gebeyehu FDRE's foreign affairs minister and all FDRE's Authority's on a top priority basis on this research abstract call. Also, I would like to express appreciation to the persons who provided support and Assistance, and deeply grateful for the dedication shown by intellectually stimulated Excellency Vice prime minister demeke mekonen.

\section{References}

1. Susmita Kamila (2013) Introduction, Classification and Applications of Smart Materials: An Overview. American Journal of Applied Sciences 10: 876-880.

2. The Manufacturer (2018) The Power of Artificial Intelligence in Manufacturing. https://www.themanufacturer.com/articles/ power-artificial-intelligence-manufacturing/.

3. Jiawei Li, Qing Xu, Neal Shah, Tim K Mackey (2019) A Machine Learning Approach for the Detection and Characterization of Illicit Drug Dealers on Instagram: Model Evaluation Study. J Med Internet Res 21: 13803.
Copyright: (02020 Amrot Mitachew Agidew. This is an open-access article distributed under the terms of the Creative Commons Attribution License, which permits unrestricted use, distribution, and reproduction in any medium, provided the original author and source are credited. 